\title{
Session 11: Novel Technology
}

Friday 16th April 2010. Moderators: Mark C. Glassy and Jim W. Larrick

[13.30-14.00]

\section{'Novel antibody functions from novel antibody} structures'

James W. Larrick, Manley Huang, Andrew Mendelsohn, Vikram Sharma, Susan C. Wright, Jianming Wang and Jeff Fang

Panorama Research Institute (PRI), Sunnyvale, California, USA

Hepatocyte growth factor (HGF) is a pleiotropic cytokine that promotes cell proliferation, motility, survival, and morphogenesis. HGF binds to its receptor c-Met tyrosine kinase and triggers signal transduction that protects cells against apoptosis and enhances cell growth for tissue regeneration. The profound effects of HGF to prevent cell death and to promote tissue regeneration make HGF an interesting drug candidate for therapeutic use. However, the activation of c-Met by HGF also lead to enhanced tumor metastasis and invasion. This pro-invasive feature of HGF has raised concerns regarding its clinical applications. It would be ideal to separate the beneficial cell-protective signals from the pro-invasive signals of HGF. To test this possibility, Michieli et al., (Nature Biotechnology 20:488; 2002) created a recombinant single-chain chimera consisting of a truncated HGF $\alpha$-chain and a truncated MSP (macrophage-stimulating protein, a cytokine with high homology to HGF) $\alpha$-chain linked by a short polypeptide linker, termed Metron factor- 1 or MF-1. This chimeric HGF-MSP protein binds to the HGF receptor c-Met and the MSP receptor Ron independently and elicits a biological signal only through Met-Ron heterodimerization. MF-1 prevents liver or renal damage and enhances liver or kidney regeneration, without inducing tumor metastasis and growth. MF-1 has a short plasma half-life (one hour) and is not suitable for development as therapeutic agent with the common i.v. or s.c. administration routes. However, it does provide a unique approach to separate the beneficial organ-protective signaling from undesired pro- invasive signals in HGF-Met pathway. Based on this work we designed and constructed a number of novel heterodimeric Fc fusion proteins ("trophokines") that can induce Met-Ron heterodimerization. Each of our novel trophokines contains an HGF-Fc and MSP-Fc fusion protein, which form heterodimers through the knobs-to-holes designed into the $\mathrm{CH} 3$ domains of their IgG1 $\mathrm{Fc}$ regions. Because of this unique design, each trophokine protein contains one c-Met binding site and one Ron binding site to induce c-Met and Ron heterodimerization. These newly designed molecules prevent target cell apoptosis and promote tissue regeneration. By eliminating the pro-invasive or scattering effects of HGF on cells, these novel proteins do not promote tumor metastasis or tumor growth. Most importantly, these novel trophokines will have significantly improved plasma half-life and will have easy manufacturing, purification, and other downstream development processes. These novel proteins are likely to provide therapeutic benefit to patients in a wide range of pathological conditions, such as liver cirrhosis, liver fibrosis, renal failure, wound healing, and chemotherapy-induced liver or renal damage. We have successfully expressed four designed trophokine fusion proteins and the results of preclinical biochemical and biological studies will be presented.

[14.00-14.30]

'Cell and vector engineering to generate highly productive cell lines'

Nic Mermod

University of Lausanne, Lausanne, Switzerland

Expression of recombinant proteins by mammalian cell lines is limited in part by transgene integration in the cell genome and by epigenetic silencing or variegation effects that decrease transcription. Epigenetic regulators such as MAR DNA elements can be used to decrease silencing, yielding cell clones with high 
and more stable transgene expression. We have found that multiple transfections of MAR-containing plasmids can promote even higher levels of transgene expression. However, this favorable effect was found to be critically dependent on parameters such as the vector, cell type, culture and transfection conditions. Multiple transfections were found to decrease targeting of the DNA to endosomal degradation and to increase transport to the nucleus. The MAR element and multiple trasnfections were found to accelerate nuclear transport and to increase the chromosomal integration of transfected DNAs. High expression levels were also influenced by the homology of the transfected DNAs and by the phasing of the cell cycle, suggesting an involvement of homologous recombination. This was confirmed by showing that $\mathrm{CHO}$ cells mutants that no longer mediate homologous recombination yielded very low expression, while $\mathrm{CHO}$ cell mutants using more prominently homologous recombination achieved the highest expression levels. Overall, we conclude that recombinant protein expression can be increased up to 25-fold when approaches combining both vector and $\mathrm{CHO}$ cell engineering are coupled to improved gene transfer methods.

\section{[14.30-14.50]}

'Development of recombinant polyclonal antibodies: implementation of new CMC analytical technologies to assess polyclonality'

Torben P. Frandsen

Symphogen A/S, Copenhagen, Denmark

Recombinant polyclonal antibody products represent a unique new class of antibody products where target specific mixtures of antibodies are manufactured as a single batch from a polyclonal cell bank. Symphogen's lead candidate (Sym001) combines 25 different $\mathrm{RhD}$ specific antibodies and is presently in phase II clinical trials. This presentation will focus on analytical methodologies that have been developed to address the specific identity of recombinant polyclonal antibody product in terms of polyclonality.

\section{[14.50-15.10]}

\section{'scFv and Fab antibody phage display libraries?'}

Isaac Rondon

XOMA, Berleley, California, USA

Therapeutic antibodies continue to gain momentum in the drug development arena due to the success rate seen with numerous antibodies moving from the lab to the clinic and into the market.|nbsp; This success has prompted many companies to enter the space of antibody based therapeutics.|nbsp; Molecular engineering methods have facilitated this success by the cloning of fully human antibodies to almost any target in a relatively short period of time.|nbsp; The development and improvements of selection and screening methods, such as phage display, yeast display, ribosomal display and others have provided means to identify specific interacting molecules from antibody libraries.|nbsp; Antibody phage display libraries are presently fueling the antibody pipelines of many companies.|nbsp; We have constructed naïve antibody phage display libraries, with scFv and Fab formats, each library having more than 2.5 e11 clones.|nbsp; These libraries have been characterized by determining open reading frame, percent expression and V-gene family distribution.|nbsp; The libraries have also been validated against a panel of targets.|nbsp; Data from the validation screening will be discussed, including differential V-gene usage per library and binding characteristics of selected clones.

\section{[15.10-15.30]}

'A new human monoclonal antibody platform based on transgenic rats'

Roland Buelow

Open Monoclonal Technology Inc., Palo Alto, CA, USA

Open Monoclonal Technology, Inc. (“OMT”, www.omtinc.net) is a private biotechnology company developing a new, fully human monoclonal antibody platform. Many companies would like to develop new monoclonal antibodies, but available technologies are expensive, and potential targets often are limited by exclusivity agreements. OMT's antibody platform is available for all targets, has broad freedom to operate, and uses technology protected by patents.

OMT's platform is based on transgenic rats. The rat is a widely used laboratory animal with a well characterized immune system, a nearly complete genome sequence, and established transgenesis and hybridoma technologies. OMT's genetic engineering approach is the result of an improved understanding of B-cell development and a novel approach to the inactivation of endogenous antibody expression. Previously, it took either embryonic stem cells or nuclear transfer cloning techniques that are not available for the genetic engineering of rats - to create a knockout. OMT developed a new zinc-finger-nuclease (ZFN)-mediated technique 
to generate immunoglobulin knockout rats. ZFNs are engineered proteins that induce double-strand breaks at specific sites in an organism's DNA. Such doublestrand breaks stimulate the cell's natural DNA-repair pathways and can result in site-specific changes in the DNA sequence. Microinjection of ZFNs specific for rat immunoglobulin $\mathrm{M}, \mathrm{V} \lambda$ or $\mathrm{C} \kappa$ into 1-cell rat embryos lead to a high frequency of animals carrying disruption at the target loci. The mutations in rat immunoglobulin caused no off-target effects in other genes, and offspring of the ZFN-edited rats carried the mutated genes. Analysis of protein expression in serum demonstrated inactivation of rat heavy and light chain immunoglobulin expression in homozygous knockout animals. Our data demonstrate that ZFNs are highly active during the earliest cell divisions in rat embryos leading to whole-animal monoallelic and, on occasion, biallelic gene disruption. ZFN-mediated gene targeting in the rat embryo is robust to changes in ZFN delivery method, concentration, and nucleic acid type, leading to rapid and highly reproducible mutagenesis of expressed and non-expressed loci in multiple inbred and outbred rat strains with a low degree of mosaicism and no observable off-target effects.

To eliminate suboptimal B-cell receptor signaling, OMT designed immunoglobulin loci including human antibody genes that allow proper assembly of the Bcell receptor complex, normal signaling and high expression of human antibodies. Immunoglobulin transgenes, encoding human kappa or lambda light chains, and a heavy chain were introduced into the rat genome using conventional embryo microinjection. Breeding of immunoglobulin transgenic and knock-out animals is ongoing and will result in the development of a new platform for generating human antibody therapeutics.

\section{[15.50-16.10]}

'The next generation of hybridoma technology'

Masahiro Tomita and Kanta Tsumoto

Mie University, Japan

Recent advances in genetic engineering now allow manipulation of target genes encoding monoclonal antibodies of interest. Affinity maturation and generation of humanized monoclonal antibodies have thereby become available. Hybridoma technology can also provide monoclonal antibodies desired against various antigens by fusing antibody-producing B lymphocytes and cancerous myeloma cells. Major advantages accrue with utilization of innate functions of immune cells and cancer cells. Conventional approaches using hemagglutinating virus of Japan (HVJ), poly(ethylene glycol) (PEG) and other practical methods based on pearl-chain formation and laser radiation, however, result in very low efficiency without selectivity because of the failure to control cell fusion. To address this problem, we have developed a new hybridoma technology termed B-cell targeting for generating novel monoclonal antibodies of interest with high efficiency and selectivity. The new approach features antigen-based pre-selection of B lymphocytes by an antigen, followed by formation of B lymphocyte and myeloma cell complexes taking advantage of the strong and specific interaction between biotin and avidin, and selective fusion of B lymphocyte-myeloma cell complexes by electrical pulses. The result is a 5-40 times higher efficiency that can be obtained with PEG-mediated methods. Recently we have further succeeded in clarifying three critical steps of B-cell targeting on the basis of immunofluorescence analysis.

Here we demonstrate that this advanced technology may be applicable to generating fully humanized monoclonal antibodies using transgenic mice, producing stereo-specific monoclonal antibodies against native structure antigens by use of antigen-expressing myeloma cells. Simultaneous production of novel monoclonal antibodies against multiple antigens is also possible, as well as short-term generation of targeted monoclonal antibodies based on in vitro immunization.

\section{[16.10-16.30]}

'Meeting the challenge of multi-transmembrane and difficult to express antigens: Hydrodynamic DNA immunization'

Robert Kelley

Genentech Inc., South San Francisco, CA, USA

Genetic immunization is an attractive approach to antibody development because native, and correctly modified (e.g. glycosylated), proteins are expressed in vivo without the need for antigen expression, production, purification and characterization. This is true not only for difficult to express proteins, but in particular, this is an attractive approach for multi-transmembrane (TM) antigens. Immunization with overexpressing (or endogenously expressing) cell lines is indeed a feasible alternative. However, if the transfected or endogenously expressing cell line is of human origin (e.g. 293, LnCap) the screening process can be extremely labor intensive and the immune response is, in general, highly non-specific. 
Here we describe the use of hydrodynamic genetic immunization, via the tail vein, for the development of antibodies against a variety of multi-TM antigens.

\section{[16.30-16.50]}

'A new platform for non-antibody protein drugs' Manuel Baca, Jeff Swers, Thomas Thisted, Lin Wang, Benoy Chacko, Luba Grinberg, Fengying Huang and Herren $\mathrm{Wu}$

MedImmune Inc., Gaithersburg, Maryland, USA

While monoclonal antibodies have enjoyed enormous success as therapeutics, there is growing interest in the development of protein drugs based on alternate scaffolds. The uptake of this technology will depend on positive outcomes in the clinic, but these new classes of protein drugs offer to combine the specificity properties of mAbs with additional advantages such as speed of discovery, ease of manufacture and format options tailored to particular disease indications. MedImmune has developed an alternate scaffold platform from a small Ig-like protein module. This $\sim 10 \mathrm{kDa}$ scaffold can be expressed at high levels in bacteria and has been engineered for high stability. Sequence and length diversification of surface loops has been used to create large diversity libraries from which target binders can be readily selected. This presentation will describe the unique features of this platform, and its application towards creating next generation therapeutics. 